\title{
Real Food for Tube Feeding Makes a Comeback
}

\author{
Wes Johnson*, Holly Carter, Teresa Johnson and Kris Maday \\ Samford University, Birmingham, AL, USA \\ *Corresponding author: Wes Johnson, Samford University, Birmingham, AL, USA \\ To Cite This Article: Wes Johnson, Holly Carter, Teresa Johnson, Kris Maday. Real Food for Tube Feeding Makes a Comeback. 2020 - 10(6). AJBSR. \\ MS.ID.001572. DOI: 10.34297/AJBSR.2020.10.001572.
}

Received: 眥 February 10,2020; Published: 眥 November 11, 2020

\section{Perspective}

Attempts to feed patients unable to eat by mouth through alternate routes are documented as early as 3500 BCE but with poor outcomes. However, successful enteral nutrition began in the 1940's with blender zed food made in hospital kitchens delivered through large bore nasogastric tubes. In the decades that followed, advances in enteral nutrition included smaller bore tubes delivering commercial formula (CF) via feeding pumps directly into the stomach or jejunum. Blenderized tube feeding (BTF) was displaced by CF in the 1960s and 70s because the latter was sterile, provided standard nutrient composition, less likely to clog small diameter tubes and covered by most medical plans $[1,2]$. Ironically, in the last two decades, health care providers (HCPs) report increasing patient interest and use of BTF to the point that formula companies are now responding by producing their own lines of whole food enteral formulas [3-11]. Despite the fact that BTF has a heavier caregiver burden, is not encouraged by HCPs and costs not routinely covered by medical plans, adherents have bought the message that highly processed food - including formula - is not an optimal feeding substrate. Conversely, HCPs trained during the years of almost exclusive CF use have expressed concerns about potential bacterial contamination, increased tube clogging, and lack of standardized nutrient profile of BTF.9,10 However, potential benefits of BTF reported by patients and their caregivers cannot be dismissed as supporting evidence continues to emerge [12].

\section{Patient and caregiver perspective}

In the era of patient-centered care and evidence-based practice, the psychosocial and safety/efficacy aspects of BTF compared to $\mathrm{CF}$ require a review of the published literature when considering BTF either as a supplement or substitute for CF. Frequently cited reasons for interest and use of BTF include the desire to provide a more physiologic feeding substrate, address tube feeding intoler ance to CF, or as a bridge to oral intake [6-11]. Patients and caregivers are favorable to BTF because it makes tube feeding less like a medical procedure and more akin to the positive experience of participating in family meals $[5,6,9]$ In addition to psycho-social benefits, BTF may be superior to CF in addressing tube feeding intolerance (e.g. reduction of gagging, retching, vomiting, diarrhea, constipation), and promoting optimal growth and weight goals [11,13-15]. Convinced of BTF safety and efficacy, users persist in this method of feeding even with little support from the medical community. In the survey of parents of tube fed children, Johnson et al found that half of BTF users relied on sources other than HCPs for tube feeding information including support groups and the Internet [6].

\section{Health care provider perspective}

Despite wanting to support the preferences of patients and caregivers regarding the use of BTF, some providers have no knowledge of it. In the 2015 survey of pediatric practice RDs, $28 \%$ of respondents indicated they needed more information on how to guide patients interested in BTF [9]. In a similar study in the United Kingdom, dietitians expressed concerns that BTF might not provide adequate nutrition, would be more likely to clog tubes, and might cause infection due to higher bacterial loads- but these were rarely observed in their clinical practices [10]. Most published studies reporting high bacterial loads of BTF were conducted in countries with very different hospital and home food preparation conditions encountered in the United States [16]. Furthermore, none of these studies correlated high bacterial loads of BTF to infections in pa- 
tients. Researchers believe high bacterial loads of enteral feeding are related more to food handling techniques than to the feeding substrate $[17,18]$. A 2018 hospital-based study in the US found BTF bacterial loads did not exceed acceptable colony forming units required of CF- even when time/temperature violations were deliberately employed during mock tube feedings [19].

Another concern of HCPs is lack of known nutrient composition of BTF compared to CF. However, this concern may be overstated given that the other family members of home enteral patients do very well on the same home-cooked meals. Furthermore, the monotony of highly processed commercial formula does not provide benefit to the gut microbiome. Research shows that diet diversity promotes a better microbiome profile and overall better health [2023]. When Gallagher et al successfully transitioned children from CF to BTF he correlated increased intestinal microbiome diversity with decreased adverse gastrointestinal (GI) symptoms and reduced need for medications for GI problems [24].

Hron et al. [25] also proposed the association between diet diversity and optimal gut microbiome diversity as a mechanism for improved outcomes in their study comparing BTF fed children to CF fed children [25]. In this prospective cohort study of 70 enterally-fed children followed for 10 months, BTF users (60\%) had significantly fewer hospitalizations and emergency department visits, higher satisfaction survey and quality of life scores, and reduced gastrointestinal symptom index scores compared to CF-fed children [25]. The researchers believe BTF was a superior feeding substrate compared to $\mathrm{CF}$ owing to a better macronutrient profile, increased viscosity, and diet diversity of a real food blend [25]. These studies challenge long-held conventional ideas by HCPs about the safety of BTF.

The decision to incorporate BTF into a patient's plan of care requires a team approach by nurses, dietitians, social workers, therapists and primary care providers. Patients and caregivers who do not feel supported by the medical community may decide to go it alone and incur risks of any tube fed patient. Only 50\% of parents currently utilizing BTF are assisted by HCPs [26]. Enteral feeding oversight is especially critical in children who have less nutrient reserves and extra nutrient demands to meet growth goals. All HCPs need to screen patients interested in or using BTF and provide referral to registered dietitians for feeding oversight and follow up. Facility policies prohibiting BTF use need to be challenged, revised and include alternative products to $\mathrm{CF}$ provided.

\section{Research Perspective}

Evidence from research studies is limited but supportive of the safety and efficacy of BTF use. Concerns that BTF cannot assure a product of known nutrient composition are not warranted due to availability of BTF resources [27-30]. Furthermore, the lack of uni- formity afforded by feeding real food makes BTF superior to the monotony of CF as demonstrated in the studies by $[15,24,25]$.

Previously stated in this paper, concerns of bacterial contamination may be overstated. Recent studies conducted in food preparation conditions expected of US homes and hospitals challenges the results of studies published in other countries showing high bacterial contamination of BTF $[16,19]$. An in-vitro study of BTF preparation conducted in food preparation conditions expected of the home setting in the US found microbial loads were considerably lower than in studies published outside the US with $88 \%$ of the samples meeting requirements for $\mathrm{CF}$ and only one sample exceeding international standards [16]. Although preparation of a sterile blended food product in a home or hospital environment is not possible, these studies demonstrate bacterial contamination risk is significantly reduced when expected food preparation procedures of US home and hospital environments are followed [16,31].

Furthermore, the concern that BTF is more likely to block tubes is not warranted when certain foods are avoided,28 high velocity blenders are used and sufficient blending times are employed. In the hospital study by [19] only one instance of a clogged tube occurred using a baby food based formula (not put through a blender), and it was easily remedied by manipulating the tube [19]. Finally, potential feeding problems of BTF are not different from CF-fed populations. Bloating, diarrhea, constipation, volume tolerance, drug/nutrient interactions, clogging, and assuring appropriate and adequate substrate are potential issues inherent with any enteral feeding and can be managed by registered dietitians in collaboration with all members of the nutrition support team. Based on current evidence, many of these problems may actually be ameliorated by BTF $[13,15,25]$.

\section{Practice perspective}

In at least six published surveys on BTF use by patients, caregivers or HCPs, up to one half of those surveyed indicated they used or recommended BTF and all reported favorable outcomes [4-6,911]. As the home enterally-fed population continues to increase $[32,33]$ the patient-driven phenomenon for demand of whole food tube feeding is bringing changes to healthcare facility policies and industry products [34-36]. More traditional formula companies are introducing whole food blend products alongside their older milk and soy-based formulas. Recommendations for BTF are included in published guidelines for nutrition support [37] and should be included in future standards of care for home enteral nutrition [38]. All HCPs- including physician's assistants should be prepared to screen enterally-fed populations for interest or use of BTF and provide referral and oversight for this feeding substrate as a part of the nutrition support team. Not all tube fed patients are candidates for BTF [28] but contraindications are waning as product development 
and safety/efficacy studies continue to show benefit across medically complex patient populations. Given the potential benefits of BTF, HCPs might consider BTF as the first option for feeding instead of standard commercial formulas.

\section{References}

1. Chernoff R (2006) An overview of tube feeding: from ancient times to the future. Nutr Clin Pract 21(4): 408-410.

2. Harkness L (2002) The history of enteral nutrition therapy: From raw eggs and nasal tubes to purified amino acids and early postoperative jejunal delivery. J Am Diet Assn 102(3): 399-404.

3. Mortensen M (2006) Blenderized tube feeding: clinical perspectives on homemade tube feeding. PNPG Post: Publ Pediatr Nutr Pract Group 17(1): 1-4.

4. Epp L, Lammert L, Vallumsetla N, Hurt RT, Mundi M (2017) Use of blenderized tube feeding in adult and pediatric home enteral nutrition patients. Nutr Clin Pract 32(2): 201-205.

5. Hurt RT, Varayil JE, Epp LM, Adele KP, Lisa ML, et al. Blenderized tube feeding use in adult home enteral nutrition patients: A cross-sectional study. Nutr Clin Pract 30(6): 824-829.

6. Johnson T, Spurlock A, Epp L, Hurt RT, Mundi M (2018) Reemergence of blended tube feeding and parent's reported experiences in their tube fed children. J Altern Complement Med 24(4): 369-373.

7. Coad J, Toft A, Lapwood S, Manning J, Hunter M, et al. (2017) Blended foods for tube-fed children: A safe and realistic option? A rapid review of the evidence. Arch Dis Child 102: 274-278.

8. Johnson T, Spurlock A, Galloway P (2013) Blenderized formula by gastrostomy tube: A case presentation and review of the literature. Top Clin Nutr 28(1): 84-92

9. Johnson TW, Spurlock A, Pierce L (2015) Survey study assessing attitudes and experiences of pediatric registered dietitians regarding blended food by gastrostomy tube feeding. Nutr Clin Pract 30(3): 402405.

10. Armstrong J, Buchanan E, Duncan H, Ross K, Gerasimidis K (2016) Dietitians' perceptions and experience of blenderised feeds for paediatric tube-feeding. Arch Dis Child 102: 152-156.

11. Kariya C, Bell K, Bellamy C, Lau J, Yee K (2019) Blenderized Tube Feeding: A Survey of Dietitians' Perspectives, Education, and Perceived Competence. Can J Diet Prac Res 80(4): 190-194.

12. Carter H, Johnson K, Johnson TW, Spurlock A (2018) Blended tube feeding prevalence, efficacy, and safety: What does the literature say? J Am Assoc Nur Pract 30(3): 150-157.

13. Gallagher K, Mouzaki M, Carpenter A, Haliburton B, Bannister L, et al. (2015) The BLEND Study: A feasibility study looking at children transitioning to blenderized tube feeds. [Supplemental Material]. Journal of Pediatric Gastroenterology and Nutrition 61: 206-207.

14. Hurt RT, Edakkanambeth VJ, Epp LM, Pattinson AK, Lammert LM, et al. (2015) Blenderized tube feeding use in adult home enteral nutrition patients: A cross-sectional study. Nutrition in Clinical Practice 30(6): 824-829.

15. Pentiuk S, O'Flaherty T, Santoro K, Willging P, Kaul A (2011) Pureed by gastrostomy tube diet improves gagging and retching in children with fundoplication. Journal of Parenteral and Enteral Nutrition 35(3): 375379.

\section{6. (JOHNSON- IN PRESS).}

17. Vieira MMC, Santos VFN, Bottoni A, Morais TB (2018) Nutritional and microbiological quality of commercial and homemade blenderized whole food enteral diets for home-based enteral nutritional therapy in adults. Clin Nutr 37(1): 177-181.

18. Baniardalan M, Sabzghabaee AM, Jalali M, Badri S (2014) Bacterial safety of commercial and handmade enteral feeds in an Iranian teaching hospital. Int J Prev Med 5(5): 604-610.

19. Johnson TW, Milton DL, Johnson K, Carter H, Hurt RT, et al. (2019) Comparison of microbial growth between commercial formula and blenderized food for tube feeding. Nutr Clin Prac 34(2): 257-263.

20. Brunkwall L, Orho-Melander M (2017) The gut microbiome as a target for prevention and treatment of hyperglycaemia in type 2 diabetes: from current human evidence to future possibilities. Diabetology 60(6): 943951.

21. Bowyer RCE, Jackson MA, Pallister T, Jane Skinner, Tim D Spector, et al. (2018) Use of dietary indices to control for diet in human gut microbiota studies. Microbiome 6(1): 77.

22. Serban DE (2015) Microbiota in Inflammatory Bowel Disease pathogenesis and therapy: Is it all about diet? Nutr Clin Prac 30(6): 760779.

23. Shanahan F, van Sinderen D, O'Toole PW, Stanton C (2017) Feeding the microbiota: transducer of nutrient signals for the host. Gut 66(9): 17091717.

24. Gallagher K, Flint A, Mouzaki M, Carpenter A, Haliburton B, et al. (2018) Blenderized Enteral Nutrition Diet Study: Feasibility, Clinical, and Microbiome Outcomes of Providing Blenderized Feeds Through a Gastric Tube in a Medically Complex Pediatric Population. JPEN J Parenter Enteral Nutr 42(6): 1046-1060.

25. Hron B, Fishman E, Lurie M, Clarke T, Chin Z, et al. (2019) Health Outcomes and Quality of Life Indices of Children Receiving Blenderized Feeds via Enteral Tube. J Peds 211: 139-145.

26. Epp L (2018) Blenderized feeding options - the sky's the limit. Pract Gastroenterol: 32-39

27. Klein M, Morris S (2007) Homemade Blended Formula Handbook. Tucson, AZ: Mealtime Notions: 1-5.

28. Walia C, Van HM, Edlbeck A, Feuling MB (2017) The Registered Dietitian Nutritionist's guide to homemade tube feeding. J Acad Nutr Diet 117(1): 11-16.

29. The Use of Blenderized Tube Feeding in Pediatric Patients: Evidence and Guidelines for Dietetic Practice. Scrib: 1-31.

30. O'Flaherty T, Santoro K, Pentiuk S (2011) Calculating and preparing a pureed-by-gastrostomy-tube (PBGT) diet for pediatric patients with retching and gagging postfundoplication. ICAN: Infant, Child, \& Adolescent Nutrition 3: 361-364.

31. Weeks C (2019) Home blenderized tube feeding: A practical guide for clinical practice. Clinical and Translational Gastroenterology 10: e-00001.

32. Mundi M, Adele P, Megan TMcM, Jacob D, Ryan TH (2017) Prevalence of home parenteral and enteral nutrition in the United States. Nutr Clin Pract 32(6): 799-805.

33. Gramlich L, Hurt RT, Jin J, Mundi MS (2018) Home Enteral Nutrition: Towards a Standard of Care. Nutrients 10(8): 1020.

34. Kathleen Z (2016) The Risks and Benefits of DIY Blended Tube Feedings. [Blog] Food \& Nutrition.

35. Bhow A, Tabrizi L, Marks C, Mencia S, Tapli A (2017) Can hospitals create a blenderized tube feeding formula that is cost effective and meets the needs of patients? Poster session presented at the annual conference of the Food \& Nutrition Conference Expo, Chicago, IL. Acad Nutr Diet 117(9)S: A9. 
36. The Growing Real Food Trend Comes to Tube Feeding

37. Boullata JI, Carrera AL, Harvey L, Escuro AA, Hudson L, et al. (2016) ASPEN Safe Practices for Enteral Nutrition Therapy. J Paren Enter Nutr 41(1): 15-103.
38. Gramlich L, Hurt RT, Jin J, Mundi MS (2018) Home Enteral Nutrition: Towards a Standard of Care. Nutrients 10(8):1020. 